\title{
Corruption, Supervision, and the Structure of Hierarchies
}

Mehmet Bac

Bilkent University

This article studies the relation between the structure of monitoring hierarchies and corruption, that is, the expected number of bribed members. The model allows for internal corruption, a form of collusion eliminating accountability (monitoring) in the hierarchy. It is shown that the number of subordinates in a corruption-minimizing hierarchy is constrained by the prospect of internal corruption and/or monitoring costs. This constraint may generate structurally independent segments. When its members are provided with the same incentive system, the middle rank of a supervision chain displays the lowest external corruption, but there is more scope for internal corruption in the upper part than in the lower part.

\section{Introduction}

Comuption is a hierarchical phenomenon. Besides the well-known example of public officials accepting bribes for a permit or licence, it includes individual opportunistic behavior in organizations, such as shirking on the job, absenteeism, and favoring friends or relatives in promotion and recruitment. Corrupt behavior can be manifest at both the individual level and at the group level, as the members can act cooperatively to organize corruption.

Despite its practical significance, few formal studies are devoted to the causes and consequences of corruption.' The literature examining the problem of motivating agents to be honest mostly takes the institutional context as given. The standard institutional context is a two-person hierarchy consisting of a principal and an agent whose actions cannot be observed, and the analysis focuses on designing and deriving properties of the efficient contractual arrangement. A

I am grateful to two anonymous referees for comments and suggestions, and Ismail Saglam for assistance. The article also benefited from a presentation at the 1994 Bosphorus Workshop on Economic Design, Marmaris, Turkey.

1. An interesting paper by Shleifer and Vishny (1993) studies economy-wide resource allocation implications of comuption. Myerson (1993) compares various election mechanisms in terms of their effectiveness in eliminating corrupt political parties. These papers address entirely different aspects of corruption than those considered here. An exception is Basu et al. (1992), who consider hierarchical chains, as in Section 3 of this paper; they work with a different set of assumptions, mainly because they do not address the problem of hierarchical design.

(C) 1996 by Oxford University Press. All rights reserved. 8756-6222/96/\$5.00 
growing literature now allows for richer organizational structures; some papers study the costs, benefits and impacts of supervision in hierarchies, while others focus on comparing the efficiency of various hierarchical structures in collecting and processing information. ${ }^{2}$ The approach in this article differs in that the economic incentives-wages, rewards and penalties-are taken as given in order to highlight the comparative effectiveness of hierarchical structures in remedying the problem of corruption.

Hierarchy is a multipurpose ordering. This ordering may reflect the transmission of information, ranking of authority delegated to the members, and the coordination mechanism within the organization. It also shows the pattern of supervision, a picture of who supervises whom. This article focuses exclusively on the supervisory role of hierarchies. I address the question, "how does the structure of a monitoring hierarchy affect the level of corruption at both the level of individual members and the group?" As expected, the answer critically depends on the technology of monitoring. I model monitoring as an activity that takes effort as input, producing a binary, random success variable. Thus, the technology is stochastic: the supervisor can never be sure that he will detect his subordinate while shirking, but he may improve the probability of detection by increasing monitoring effort. Among alternative ways of extending these basic features to the case of many subordinates, I choose two rather natural, polar extensions: public monitoring and private monitoring. As their names suggest, under public monitoring, a given monitoring effort induces the same probability of detection on all the subordinates, while the private monitoring technology stipulates separate, individual monitoring of each subordinate.

Besides the technology used in monitoring, another relevant issue is the nature of corruption. Viewed as an organizational phenomenon, corruption is usefully decomposed along two dimensions. The first dimension is what I call external corruption, referring to transactions between a member of the organization and an outsider. A bribe-taking official is the typical example of external corruption. The second dimension is intermal corruption, a "violation of duty" that results from collusive behavior within the organization. Internal corruption can take the form of an implicit agreement, whereby the subordinates transfer a portion of the proceeds from external corruption (bribes) to the upper levels and possibly obtain nonpecuniary benefits and protection in return. Clearly, internal corruption banishes the supervisory function of the hierarchy. This type of corruption is often reported to exist within the police organizations of developing countries. Jabbra (1989), for instance, reports how public officials in Lebanon devised a trick to beat the system introduced to remedy the problem of absenteeism: the officials simply alternated in punching each other's time

2. Early examples of the literature on the supervisory role of hierarchies are Williamson (1967), Calvo and Wellisz (1978), and Calvo (1987). See also Varian (1990). Examples of the second line of research include Radner (1993), who studies the optimal structure of a hierarchy that minimizes delay in processing items and communicating information. Baron and Besanko (1992) compare the performances of hierarchical contracts in maximizing a regulator's surplus when the regulato lacks the relevant information possessed by the agents. Another paper in this vein is Bolton and Dewatripont (1994). 
cards or asked their janitors to do it. Overcentralization and overstaffing are among the most cited reasons of internal corruption in the informal public administration literature. The internal dimensions of corrupt behavior have long been an active field of research in the informal organization literature (e.g., Cyert and March, 1963). The issue has only recently been recognized in the economics literature and studied in formal models, to my knowledge, by Tirole (1986, 1988).

The analysis sheds light on the relation between the structure of hierarchies, the monitoring technology, and the possibilities of internal and external corruption. It identifies a trade-off between external corruption and the risk of internal corruption in the design of hierarchies. The trade-off depends critically on the technology used in monitoring the subordinates. Generally, for any population size $N$, the number of subordinates per supervisor is constrained by the possibility of internal corruption. This constraint, however, may or may not be binding, depending on the size of the population, the monitoring technology, and other variables affecting the supervisor's net expected private benefit from monitoring. It is most likely to be binding under the public monitoring technology, because under this technology, the total cost of monitoring does not depend on the number of subordinates. Economizing on monitoring costs may require flat and centralized hierarchies, but the need to keep internal corruption under control calls for decentralization. If the size of the population is large, internal corruption considerations may thus generate hierarchical organizations that are structurally independent.

While the public monitoring technology favors flat hierarchies, the private monitoring technology favors rather steep monitoring structures. Such steep hierarchies limit the span of supervision and are relatively immune to internal corruption, because the potential size of corrupt proceeds that can be used in bribing the supervisor is small. Examining a three-rank hierarchy, where the members are provided with an identical incentive system, I find that in the noncooperative outcome, the middle-rank agent is the least likely to accept a bribe from an outsider. Adding a third rank to the hierarchy actually increases the bottom-rank agent's expected corruption. On the other hand, the upper part of this hierarchy (the top and the middle agent) displays a higher risk of internal corruption than its lower part (the middle and the bottom agent).

The article is organized as follows. The next section introduces the model and studies the impact of hierarchy in the case of two agents. Section 3 introduces a third agent and looks at the equilibrium of the resulting supervision chain. Section 4 provides definitions of various hierarchies and monitoring technologies that are later used in the analysis of Sections 5 and 6 . Section 7 contains a discussion of results and extensions.

\section{The Basic Model}

Consider a principal and a group of $N$ identical risk-neutral agents who are paid the wage $w$ for a service. $w$ can be thought of as a reservation wage. ${ }^{3} N$

3. The most common theoretical justification for flat wages is the presence of severe problems in measuring and evaluating performance, and the resulting noncontractibility of the agents' output 
is assumed finite throughout the article. The agents enjoy some discretion in their job, which they may misuse for personal benefit. As a way of representing this potential for extemal corruption, I let each agent in the model be offered a "bribe" of $z$ dollars. The agent either accepts $\left(b_{i}=1\right)$ or rejects $\left(b_{i}=0\right)$ the bribe. For example, we can think of the agents as officials pledged to produce public services, and of the principal as the government. There is no need to interpret the bribe in this context. Alternatively, the agents may be the employees of a firm. The firm may not be able to directly observe the product and effort of its employees, and therefore would resort to active monitoring and layoffs to ensure a minimum acceptable performance. In this context, the bribe $z$ can be interpreted as the value of on-the-job consumption. The bribe should thus be understood in a broad sense, meaning any private benefit the agents reap through "violation of duty."

To simplify the analysis, I assume that it is completely safe to take bribes in the absence of monitoring. The expected utility and participation constraint of agent $i$ in the absence of monitoring is

$$
E U_{i}=b_{i}(w+z)+\left(1-b_{\imath}\right) w \geq w,
$$

which, not surprisingly, shows that accepting the bribe is the dominant strategy of each agent.

As mentioned in Section 1, the analysis focuses on the impact of hierarchical monitoring arrangements on corruption. In other words, it aims at characterizing the hierarchical monitoring structure that minimizes the expected number of agents who accept bribes, $\sum_{i} b_{i}$, taking as given the set of economic incentives. By hierarchical monitoring, we mean a set of antisymmetric monitoring relations implied naturally by the structure of the hierarchy. ${ }^{4}$ For instance, we say that agent $A$ is one rank above agent $B$ if $A$ monitors $B ; A$ is two ranks above $B$ if $A$ monitors someone who monitors $B$, and so on. Peer monitoring is ruled out to focus on the hierarchical dimension. Assumptions about the private costs and benefits of monitoring are presented below.

or service. Casual observations support the view that wage structures are related to contractibility. Salesmen whose output is easily measurable and contractible mostly receive piece rates, while civil servants in public organizations have fixed wages. The lack of goal clarity in public organizations is a major contributor to the problem of measuring output. For instance, it is not clear whether the output of a police department should be measured by the number of arrests, convictions, calls responded to, or family disputes resolved. See Hart and Holmstróm (1987) for a comprehensive discussion of contractual arrangements.

4. An implication of this assumption is that top-level supervisors will not be monitored and thus will accept the bribe. Of course, in general, it is possible to provide incentives to a supervisor by giving him the residual claimant status over the output of his subordinates (Alchian and Demsetz, 1972). There are circumstances, as the public office example mentioned above, where such residual claims cannot be provided. For a discussion and examples of the consequences of ill-specified property rights in Soviet-type economies, see Major (1993). Another mechanism for supervision is the use of outside investigators. This mechanism is not considered here because it does not seem to be qualitatively relevant for our results. Having outside parties detect corruption with some probability in our model will reduce overall corruption but it does not affect the ranking of hierarchies in terms of displayed corruption. 


\subsection{The Benchmark Hierarchy}

It is useful to study first the simplest case of $N=2$. Suppose agent $A$ is the supervisor and agent $B$ is the subordinate. Recall that the agents are identical in all respects, hence, only their hierarchical positions matter. In the following sections, I refer back to this simple structure as the benchmark hierarchy. The agents' set of opportunities is as described above, except that now monitoring is included in the picture. Let $p$ denote the private benefit that $A$ will obtain if he successfully proves and reports $B$ 's corrupt activities. Throughout the analysis, $p$ is exogenous: it can be a monetary reward or the value of improved career expectations, or any improvement in $A$ 's utility in successfully monitoring his subordinate. ${ }^{5}$ If $A$ monitors successfully and so proves that $B$ is bribed, then $B$ receives a utility normalized to zero (say, because he is fired).

Monitoring costs are described by the function $c(m)$, where $m$ is the monitoring effort. Success is stochastic: the bribe is detected in a way that can be verified by third parties with probability $\mu(m)$. If $B$ is bribed, $B$ 's income is therefore $w+z$ with probability $1-\mu(m)$, and zero with probability $\mu(m)$. In Section 4, I extend this monitoring technology to accommodate the monitoring of many subordinates. I make the following assumption.

Assumption 1. The cost function $c: R_{+} \rightarrow R_{+}$is increasing and strictly convex, and $\mu: R_{+} \rightarrow[0,1]$ is increasing and strictly concave with $\mu(m) \rightarrow 1$ as $m \rightarrow \infty$. Furthermore, $\partial \mu(m) / \partial m \rightarrow \infty$ and $\partial c(m) / \partial m \rightarrow 0$ as $m \rightarrow 0$.

The expected utilities of $A$ and $B$ can be written as follows.

$$
E U_{A}=b_{A}\left[w+z+\mu(m) b_{B} p\right]+\left(1-b_{A}\right)\left[w+\mu(m) b_{B} p-c(m)\right.
$$

and

$$
E U_{B}=b_{B}[1-\mu(m)](w+z)+\left(1-b_{B}\right) w .
$$

Since $A$ is not monitored and $z>0$, from (2) it follows that $b_{A}^{*}=1$. Introducing a simple monitoring hierarchy creates a game situation between $A$ and $B$, where the parties' strategies are $m$ and $b_{B}$, respectively. A Nash (noncooperative) equilibrium of this game is defined as a monitoring strategy $m^{*} \in[0, \infty]$ and a possibly mixed strategy of bribe acceptance $b_{B}^{*} \in[0,1]$, such that $m^{*}$ maximizes (2) given $b_{B}^{*}$, and $b_{B}^{*}$ maximizes (3) given $m^{*}$. Equilibrium monitoring and the probability of bribe acceptance are always strictly positive due to Assumption 1. It will be useful, however, to draw the following distinction between potential types of equilibria: an equilibrium in completely mixed bribe acceptance strategies $b_{B}^{*} \in(0,1)$ is called a type-l equilibrium. It

5. $p$ can be interpreted in two ways. Besides its interpretation as the prize awarded by the principal to each successful bribe report, $p$ can be thought of as a cost incurred by the supervisor who is not able to (or deliberately does not) document bribed subordinates. This second interpretation can be used, if we add an exogenous probability of bribe detection by "outsiders," so that the supervisor can be punished for not reporting, if his subordinate is proved corrupt by outside investigators. With this modification, the supervisors' decision-making process is similar under both interpretations; we carry the analysis in terms of the analytically simpler, first interpretation. 


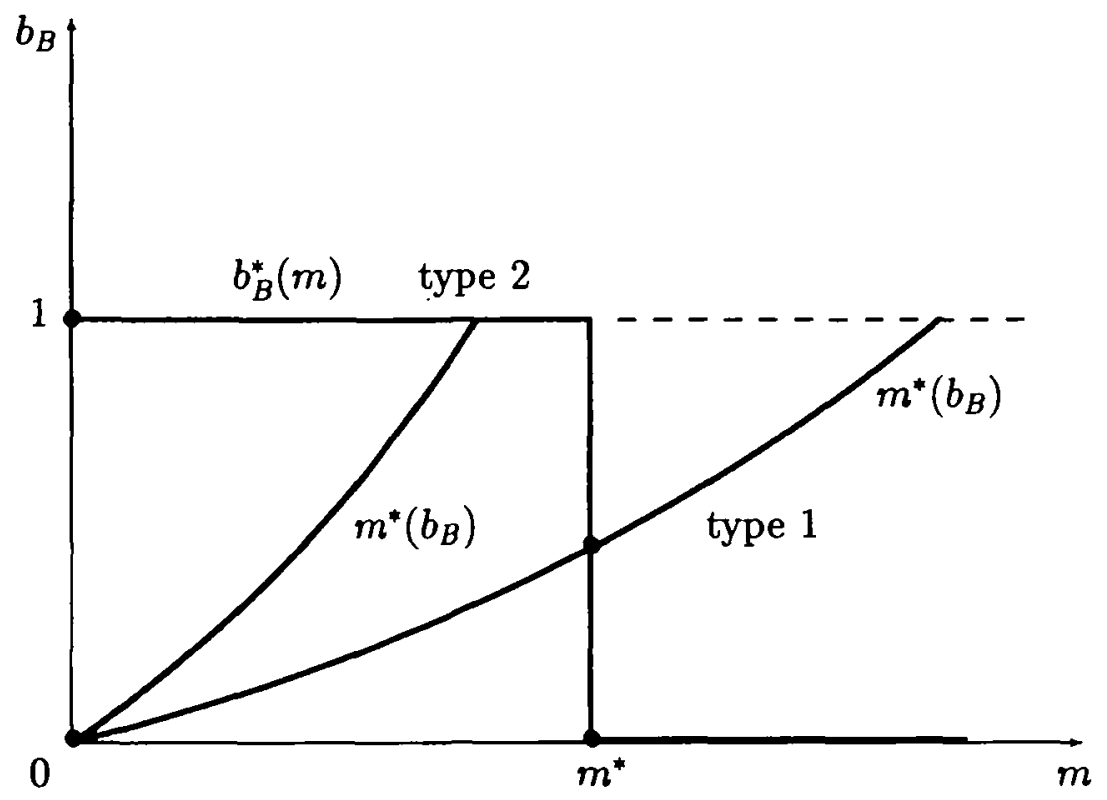

Figure 1. Type-1 and type-2 equilibria in the benchmark hierarchy

is characterized by the two conditions that follow.

$1-\mu\left(m^{*}\right)=w /(w+z)$.

$p b_{B}^{*}=\frac{\partial c\left(m^{*}\right) / \partial m}{\partial \mu\left(m^{*}\right) / \partial m}$.

On the other hand, type-2 equilibria may arise if monitoring costs increase sharply in effort and/or the success probability is sufficiently inelastic. This type of equilibria involves a comer solution $b_{B}^{*}=1$ with a lower monitoring effort ( $m<m^{*}$ ). Condition (4) must therefore be adjusted as $1-\mu(m)>w /(w+z)$, but the condition in (5) for optimality of monitoring effort keeps holding for $b_{B}^{*}=1$. These two types of equilibria are illustrated in Figure 1, where $b^{*}(m)$ and $m^{*}(b)$ represent, respectively, $B$ 's and $A$ 's best-reply functions.

These equilibria differ in an important respect, that is, in whether or not monitoring is effective in reducing expected corruption. In a type-2 Nash equilibrium, induced monitoring is ineffective; it is too low to have an impact on subordinates who then surely accept the bribes. In a type-1 equilibrium, however, the subordinate hesitates. Unless stated otherwise, I assume that monitoring is effective in order to focus on the analytically more interesting case of type-1 equilibria.

Assumption 2. In the Nash equilibrium of the benchmark hierarchy, the subordinate's bribe acceptance strategy is completely mixed, that is, $b_{B}^{*} \in(0,1)$. 
Besides the impacts of $c(\cdot)$ and $\mu(\cdot)$ mentioned above, (3) and (5) show that Assumption 2 is more likely to hold as $w$ and/or $p$ is increased or $z$ is decreased. In a type-1 equilibrium, $A$ 's monitoring $m^{*}$ has an impact on $B$ 's behavior, hence, the monitoring hierarchy is functional. Condition (4) states $B$ 's indifference between accepting and rejecting the bribe. Assumption 1 ensures that the Nash equilibrium $\left(m^{*}, b_{B}^{*}\right)$ exists and is unique, and under Assumption 2, we have $m^{*}\left(b_{B}\right)>m^{*}$ whenever $b_{B}=1$. On the other hand, $b_{B}=0$ cannot be an equilibrium strategy, because $A$ would then stop monitoring, which, in turn, would induce $B$ to set $b_{B}=1$. Hence, $b_{B}^{*} \in(0,1)$. The participation constraints can be verified using (2) and (3); they simplify to $z+\mu\left(m^{*}\right) b_{B}^{*} p-$ $c\left(m^{*}\right) \geq 0$ for agent $A$, and $\left[1-\mu\left(m^{*}\right)\right] z-\mu\left(m^{*}\right) w \geq 0$ for agent $B$. While $B$ 's participation constraint is binding, $A$ 's is never binding, because he expects a nonnegative private retum $\mu\left(m^{*}\right) b_{B}^{*} p-c\left(m^{*}\right) \geq 0$ from monitoring, plus the bribe $z$. As I show in the following sections, in a type-1 equilibrium, bottom-rank agents' participation constraints will always be binding, no matter the hierarchical form. I will frequently refer back to conditions (4) and (5) when characterizing the noncooperative type-1 equilibrium $\left(m^{*}, b_{B}^{*}\right)$ as the equilibrium outcome of the benchmark hierarchy.

Consider now the possibility of internal corruption, that is, the members' cooperation aimed at eliminating the supervisory function of the hierarchy. The potential for mutually beneficial arrangements between $A$ and $B$ arises in two occasions, before and after $A$ monitors $B$, because $B$ can pay $A$ to avoid being monitored and to avoid being reported if caught taking the bribe. I assume that the parties will collude whenever net expected benefits from collusion are positive. ${ }^{6}$ To derive the conditions under which collusion does not occur, recall the equilibrium condition (4). Used in (3), it implies that $B$ 's expected income is the wage $w$. Since taking the bribe can yield the surplus $z$ to $B, z$ is the maximum that $B$ would pay $A$ to avoid being monitored. Hence, there is room for collusion, unless

$\mu\left(m^{*}\right) b_{B}^{*} p-c\left(m^{*}\right) \geq z$.

Condition (6) shows that the expected gross benefit $\mu\left(m^{*}\right) b_{B}^{*} p$ from monitoring, hence also the "prize" $p$ from successfully reporting the bribe, must exceed the bribe offered to $B .^{7}$ In the other occasion for collusion, $B$ is caught taking the bribe. $B$ would be willing to bribe $A$ up to $w+z$ to avoid being reported. Hence, for $A$ to report bribing, $p \geq w+z$, but as one would expect,

6. Note that this rules out the costs of negotiating and enforcing implicit collusions, a particularly restrictive assumption when the number of negotiating agents becomes large. Obviously, the presence of negotiation costs can mitigate internal corruption. The extension of this basic model to a dynamic, repeated setting can generate an endogenous no-collusion constraint in the spirit of the folk theorem; if the agents are sufficiently patient and farsighted, implicit collusion will be self-enforcing. Pechlivanos (1995) examines this issue in a repeated principal-agent-client model in which the principal controls the environment where the agent and the client interact.

7. Of course, this does not mean that $B$ should not be monitored, because the social cost of the bribe may be larger. It is, nevertheless, suggestive of the high cost of reducing corruption in public offices through economic incentives. 
this is already implied by (6). Hence, $A$ does not collude ex post with $B$ if he does not collude ex ante with $B$.

\subsection{Discussion of the Assumptions}

The prime motivation for assuming an exogenous incentive system $(w, p)$ is that identical wages for all agents and identical reward structures for all supervisors will better highlight the pure effect of the hierarchical structure on corruption. One can easily see the pure effect of adding a third rank to the benchmark hierarchy or of increasing the number of subordinates. Needless to say, this assumption also simplifies the analysis. Another motivation stems from the fact that hierarchies are multipurpose structures performing much more than the supervisory function considered here; these functions should also be reflected in the incentive system. A theory of incentives and wage determination based exclusively on the supervisory function in a potentially corrupt hierarchy will thus inevitably be incomplete and possibly misleading. Furthermore, endogenizing $(w, p)$ requires specification of an objective according to which the organization's benefit from reduced cormution will be evaluated against the corresponding monetary cost of incentives; this specification necessarily involves some arbitrariness, which we prefer to avoid in this article. For instance, if the objective is to minimize a function $\Gamma\left(\sum b_{i}\right)$ of the expected number of bribes plus the monetary cost of incentives, then the results depend crucially on the specification of $\Gamma$. Introducing a budget constraint for the monetary cost of incentives would further complicate the problem. One can also allow for monitoring technologies that differ according to the supervisor's position or rank, or consider monitoring of not just the immediate subordinates but also several layers down the hierarchy. Despite these difficulties in studying more complex hierarchies, the extension to fully endogenize $w_{i}$ and $p$ within multipurpose hierarchies is obviously worth exploring in subsequent research. ${ }^{8}$

The results presented below, then, are obtained under the assumptions outlined in Section 2.1: all agents receive the same wage $w$, and all supervisors face the same monitoring incentive, a reward $p$ per successful bribe report. Penalties and rewards are bounded. Given this incentive scheme, we focus on the impact of hierarchical structure and monitoring technology on the corruption level. We shall, however, comment on the potential impact of endogenizing the incentive system whenever possible.

\section{The Supervision Chain with $N=3$}

The analysis of the supervision chain of three agents will provide intuition for factors affecting the shape of corruption-minimizing hierarchies with large populations. In this section, I study a supervision chain where agent 1 monitors agent 2 with an effort $m_{1}$, and agent 2 in turn monitors agent 3 with an effort $m_{2}$. There is now a new element in the analysis: a middle-rank member who

8. Bac (1996) takes a step in this direction for the case $N=3$ and compares the cost of implementing a target level of corruption under alternative hierarchical structures. 
supervises and is supervised at the same time. The agents' objective functions are

$$
E U_{1}=w+z+\mu\left(m_{1}\right) b_{2} p-c\left(m_{1}\right)
$$

for agent 1 , and

$$
\begin{aligned}
E U_{2}= & {\left[1-\mu\left(m_{1}\right)\right] b_{2}\left[w+z+p b_{3} \mu\left(m_{2}\right)\right] } \\
& +\left(1-b_{2}\right)\left[w+p b_{3} \mu\left(m_{2}\right)\right]-c\left(m_{2}\right)
\end{aligned}
$$

for agent 2. Agent 3's objective is as given in (3). The expression for $E U_{1}$ includes the information $b_{1}^{*}=1$, because agent 1 is not monitored. The expression for $E U_{2}$ takes the impact of agent 1's monitoring into account: if agent 2 accepts the bribe, he expects an income of $w+z+p b_{3} \mu\left(m_{2}\right)$ only if he himself is not caught, which happens with probability $1-\mu\left(m_{1}\right)$. He thus receives a zero income with probability $\mu\left(m_{1}\right) b_{2}$. On the other hand, if he is honest, his expected income is $w+p b_{3} \mu\left(m_{2}\right)$. Note that agent 1 's monitoring affects the net benefit agent 2 expects from monitoring agent 3 .

The Nash equilibrium consists of a monitoring and a bribe acceptance strategy ( $\left.m_{i}^{*}, b_{i}^{*}\right)$ maximizing $E U_{i}$ for each $i=1,2,3$, given $\left(m_{-i}^{*}, b_{-i}^{*}\right)$. To show the impact of introducing a third rank to our benchmark hierarchy, I compare below the equilibrium bribe acceptance strategies $b_{2}^{*}$ and $b_{3}^{*}$ with $b_{B}^{*}$.

Proposition 1. In the absence of internal corruption, and given the same incentive system $(w, p)$ in a hierarchical chain of three agents, a monitored monitor is less likely to be bribed than a monitored nonmonitor: $b_{3}^{*} \geq b_{B}^{*} \geq b_{2}^{*}$.

Proof. Let Assumption 2 hold so that $b_{B}^{*} \in(0,1)$, and let $b_{2}^{*} \in(0,1)$ and $b_{3}^{*} \in(0,1)$. Agent 3's indifference about $b_{3}=0$ and $b_{3}=1$ means that $1-\mu\left(m_{2}^{*}\right)=w /[w+z]$. Therefore, $m_{2}^{*}=m^{*}$, where $m^{*}$ is the monitoring effort in the benchmark case of Section 2 . On the other hand, $m_{2}^{*}$ must be optimal for agent 2 , as expressed in the first-order condition (7).

$\left[1-b_{2}^{*} \mu\left(m_{1}^{*}\right)\right] p b_{3}^{*}=\frac{\partial c\left(m_{2}^{*}\right) / \partial m_{2}}{\partial \mu\left(m_{2}^{*}\right) / \partial m_{2}}$.

Notice that the right-hand sides of (7) and (5) are identical, hence, from (7) it follows that $b_{3}^{*}>b_{B}^{*}$. (Obviously, if the equilibrium is of type 2 so that $b_{B}^{*}=1$, then $b_{3}^{*}=1$ as well.) As for the monitoring effort of agent $1, m_{1}^{*}$ satisfies the following first-order condition:

$p b_{2}^{*}=\frac{\partial c\left(m_{1}^{*}\right) / \partial m_{1}}{\partial \mu\left(m_{1}^{*}\right) / \partial m_{1}}$.

$b_{2}^{*} \in(0,1)$ means that agent 2 is indifferent about accepting and rejecting the bribe, which, using $E U_{2}$, can be expressed as

$$
1-\mu\left(m_{1}^{*}\right)=\frac{w+p b_{3}^{*} \mu\left(m_{2}^{*}\right)}{w+z+p b_{3}^{*} \mu\left(m_{2}^{*}\right)}
$$

Equation (9) shows that $m_{1}^{*}<m_{2}^{*}$, hence, from (8) and (5), we get $b_{2}^{*}<b_{B}^{*}$. 
Supervision has more impact on supervisors than on bottom-rank subordinates. In this supervision chain of three agents who all receive the same wage $w$ and are given the same prize $p$ per bribe report for the supervisors, agent 2 is the least likely to get bribed. This is so because agent 2 has more to lose, compared to agent 3, when he is caught accepting the bribe. Even if agent 2 successfully monitors and has proof that agent 3 is corrupt, his own corruption may be detected by agent 1 , in which case agent 3 and agent 2 are penalized, and their incomes fall to zero. As a result, agent 2 modifies his behavior. $\mathrm{He}$ is less inclined to accept the bribe and monitors with a lower effort, which in turn makes agent 3 more inclined to accept the bribe. 9,10

Consider now the possibilities for internal corruption in this three-rank hierarchy. Because the participation constraints of agent 2 and agent 3 are binding, each subordinate is willing to bribe his supervisor up to $z$ to avoid being monitored. Agent 1 will reject a bribe of $z$ from agent 2 if

$\mu\left(m_{1}^{*}\right) b_{2}^{*} p-c\left(m_{1}^{*}\right) \geq z$,

and, similarly, agent 2 will reject agent 3's offer of $z$ if

$\left[1-\mu\left(m_{1}^{*}\right) b_{2}^{*}\right] p b_{3}^{*} \mu\left(m_{2}^{*}\right)-c\left(m_{2}^{*}\right) \geq z$.

Equations (10) and (11) are thus the no-collusion constraints. Clearly, agent 2 must be implied in any collusion because he has monitoring relations with the other two agents. Comparing (10) and (11) reveals which part of the hierarchy is more likely to exhibit internal corruption.

Proposition 2. In the supervision chain of three identical agents facing the incentive system $(w, p)$, the case for collusion between agent 1 and agent 2 is stronger than between agent 2 and agent 3 .

Proof. Using (5) and the fact that $m_{2}^{*}=m^{*}$, (7) can be written as [1 $\left.b_{2}^{*} \mu\left(m_{1}^{*}\right)\right] p b_{3}^{*}=p b_{B}^{*}$. This in tum implies that the no-collusion constraint (11) between 2 and 3 is equivalent to (6), provided that (10) holds. Hence, if 1 and 2 do not collude, the incentives for internal corruption in the lower part of the hierarchy are identical to those in the benchmark hierarchy. Now assume that (10) holds. Given the fact that $m_{1}^{*}<m^{*}$ and $b_{2}^{*}<b_{B}^{*}$, and from the properties of $c(\cdot)$ and $\mu(\cdot)$ listed in Assumption 1, it follows that $\max _{m}\left\{\mu(m) b_{B}^{*} p-c(m)\right\}>\max _{m}\left\{\mu(m) b_{2}^{*} p-c(m)\right\} \geq z$. Using in these inequalities the fact that $\left[1-b_{2}^{*} \mu\left(m_{1}^{*}\right)\right] p b_{3}^{*}=p b_{B}^{*}$ shows that (11) is implied by (10).

9. Given the result in Proposition 1, the principal may increase the prize $p$ for agent 2 's successful bribe report and decrease agent 1 's $p$ in order to reduce the gap between $b_{2}^{*}$ and $b_{3}^{*}$. Whether this is beneficial or not, of course, all depends on the principal's objective, as mentioned in Section 2.2, and on the curvatures of $c(\cdot)$ and $\mu(\cdot)$.

10. See Basu et al. (1992) for an alternative analysis of hiesarchical chains, where taking bribes from a client occurs only at the bottom layer, and the probability of detection is exogenous. They assume that the captor gets no reward from reporing the bribe and use the Nash bargaining solution to determine the distribution of the gains from collusion. 
It is possible to have an outcome where only agent 1 and agent 2 collude, but a collusion that leaves out agent 1 or agent 2 is impossible. Since the no-collusion constraints (10) and (11) are derived from the noncooperative equilibrium strategies, recalling Proposition 1 will be helpful in understanding the intuition behind this result. In the noncooperative equilibrium, agent 1 's net expected benefit from monitoring is lower than agent 2's, because agent 2 is less inclined to accept the bribe than agent 3 . Since each subordinate is at most willing to offer the same bribe $z$ to avoid being monitored, which of the two collusion-proof constraints is more likely to be violated all depends on the expected rewards of the two supervisors. It is obviously easier to collude with a supervisor when his expected benefit from monitoring is low. Therefore, agent 2 needs less resources to stop agent 1 from monitoring than agent 3 needs in order to stop agent 2 . The risk of internal corruption is thus higher at the top than at the bottom of the hierarchy. If only agent 1 and agent 2 collude, the lower part of the hierarchy has the Nash outcome of the benchmark case, and the corresponding strategies are $b_{1}=b_{2}=1, b_{3}=b_{B}^{*}$, and $m_{2}^{*}=m^{*}$.

I have thus far described the basic features of the model and studied the outcomes of the benchmark hierarchy consisting of two agents, and of the supervision chain of three agents. I proceed with a discussion of alternative ways of introducing hierarchies with monitoring possibilities for the general case of $N$ agents.

\section{Hierarchical Extensions and Monitoring Technologies}

There are a great many possible divisions of a large group of people into smaller groups; hence, a great many hierarchical structures are possible. The steepest hierarchy for any group of agents would be complete ranking of the agents, while a very flat ranking would be one that had one leader supervising everyone else. In addition to such characteristics as steepness, hierarchies may be composed of various structures, from one that is unitary to others that involve mutually independent segments. In the limit case, the number of independent segments is maximum when each agent is an independent unit on its own; one can think of this extreme case as the flattest possible hierarchy-where in fact there is no hierarchy in the sense in which hierarchy is usually understood.

As mentioned, the pattern of monitoring corresponds to the hierarchical structure. The supervisor can monitor the subordinate, but a subordinate cannot monitor his supervisor. The span of monitoring is well-defined in the sense that no agent is assigned to more than one supervisor, but a supervisor can obviously monitor many agents. In the following sections, I compare various hierarchies in terms of the "expected number of agents who accept bribes," a proxy for the expected level of comption in the hierarchy. A systematic way of building hierarchies from a given population is through vertical extensions of the population, that is, by generating ranks. The definitions of some important extensions are in order below.

A minimal one-rank extension of the population brings one agent (the supervisor) to the top, with all other $N-1$ agents remaining at the bottom. This is the flattest possible one-rank extension of the population, hence, the flattest 


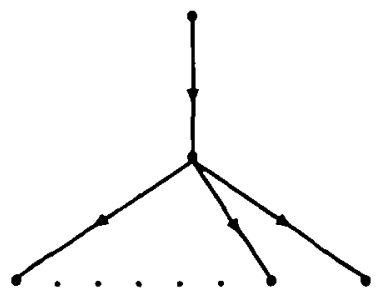

Minimal Two-rank Extension
1

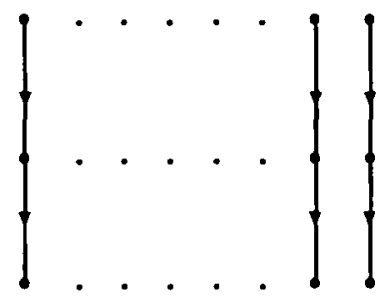

Maximal Two-rank Extension

Figure 2. Minimal and maximal two-rank extensions of the population.

possible two-rank hierarchy. A maximal one-rank extension of the population consists of dividing the population into two groups of sizes as equal as possible, with the smaller of the two groups forming the higher rank, the supervisors. If $N$ is odd, $(N-1) / 2$ are at the top rank and $(N+1) / 2$ are at the bottom rank. Depending on the costs and benefits of monitoring, it may be optimal either to leave aside one agent and form $(N-1) / 2$ supervisor-subordinate pairs, or to monitor the residual agent through one of the supervisors. If $N$ is even, however, each subordinate is matched with one supervisor. This extension generates $N / 2$ independent segments, with each segment consisting of two agents. Maximal and minimal $k$-rank extensions can be defined similarly. To exemplify, the minimal and maximal two-rank extensions are presented, respectively, on the left and the right side of Figure 2. Other intermediate extensions and specific types of hierarchies that include independent segments will be defined later.

The technology of monitoring, that is, the relationship between total costs of monitoring and the likelihood of success in monitoring each one of the subordinates, is an important element of the model. To proceed further in the analysis, I extend the definition of the monitoring technology to accommodate the case in which many subordinates are monitored by one supervisor. I shall consider the following two polar technologies: (i) public monitoring, where, given the supervisor's total monitoring effort $m$, the probability of successfully monitoring each subordinate is $\mu(m)$, no matter the number of subordinates, and (ii) private monitoring, where the effort used in monitoring a particular subordinate has no impact on the probability of successfully monitoring another subordinate.

The public monitoring technology is more representative of situations where a group of subordinates are all simultaneously "visible" to the monitor. The effort $m$ generates the probability $\mu(m)$ of successfully monitoring the entire group of subordinates, thus implying decreasing per-subordinate monitoring costs. Monitoring is "public" in that the supervisor cannot exclude a subset of subordinates or monitor them less intensively than another set of subordinates. "

11. Though the definition is extreme in its assumption that monitoring costs do not depend on the number of subordinates, it may provide a good approximation for cases in which the number 
Prima facie, with public monitoring, one expects the level of corruption to be lowest in the minimal one-rank extension for two reasons. First, per-subordinate costs of monitoring decline with the number of subordinates for a given probability of success. Second, all agents except one (the supervisor), are monitored. On the other hand, under private monitoring, each subordinate has to be monitored separately. As a result, letting $\mathrm{km}$ be the total monitoring effort, and assuming that the supervisor allocates this effort equally among the $k$ subordinates, the probability of successfully monitoring each subordinate will be $\mu(m)$. But effort is $k$ times higher, and costs are more than $k$ times higher than under public monitoring. Thus, the major difference between the two technologies lies in the impact of the number of subordinates on monitoring costs. Note that since $c(\cdot)$ is strictly convex, $c(k m)>k c(m)$ for all integers $k>1$, which implies rising per-subordinate costs under private monitoring. This monitoring technology is thus more representative of situations where simultaneous monitoring of more than one subordinate by one supervisor is prohibitively costly.

I proceed with the study of two polar one-rank extensions of the population under public and private monitoring, respectively. To facilitate the comparison with the benchmark hierarchy, I maintain the assumption that the reward to each successful bribe report is $p$.

\section{Public Monitoring}

5.1 The Minimal One-Rank Extensıon

The hierarchy resulting from the minimal one-rank extension has only one supervisor monitoring the remaining $N-1$ agents. The supervisor chooses $b^{*}=1$ and $m$ to maximize (2), where $b_{B}$ should be replaced by $\sum_{t} b_{i}$. (If monitoring is successful, the supervisor can detect an expected number $\sum_{i} b_{i}^{*}$ of bribes.) Each subordinate maximizes (3), where $b_{1}$ should replace $b_{B}$. With these similarities in the objectives, we have good reasons to suspect that the Nash equilibria of the minimal one-rank extension and the benchmark hierarchy will also have similar properties. In a type- 1 equilibrium, the supervisor will monitor with an effort that makes each subordinate indifferent about taking the bribe. The indifference condition $b_{i}^{*} \in[0,1]$ is stated in (4), and it holds for a unique monitoring effort $m^{*}$. This $m^{*}$ and the $b_{i}^{*}$ now satisfy (12):

$p \sum_{i} b_{i}^{*}=\frac{\partial c\left(m^{*}\right) / \partial m}{\partial \mu\left(m^{*}\right) / \partial m}$.

Equation (12) is a restatement of (5), except that the sum of $b_{i}^{*}$ over $N-1$ subordinates replaces $b_{B}^{*}$. Observe that the right-hand sides of (5) and (12) are equal, because $m^{*}$ is identical in both expressions. Hence, their left-hand sides, too, must be equal. It follows that given the benefit $p$ expected from successfully reporting a detected bribe, we have $\sum_{i} b_{i}^{*}=b_{B}^{*}$ in equilibrium.

of subordinates is relatively small. This independence assumption not only simplifies the analysis and sets up a useful benchmark, but as shown in the following sections, it also clears the way for another factor that limits the span of supervision, the possibility of internal corruption. 
With $N-1$ subordinates and public monitoring, on average, it is $N-1$ times less likely that each subordinate is bribed with respect to the single subordinate case. Therefore, the sum of Nash equilibrium bribe acceptance probabilities of the members, or the expected number of bribes, is $\Pi_{m}=1+\sum_{1} b_{i}^{*}$. This number is less than two no matter the population size $N$. Note also that the expected cost of the linear reward system is independent of the number of subordinates, as $p \sum b_{i}^{*}$ is constant and equal to $p b_{B}^{*}$. Therefore, the expected cost of rewards does not depend on the reward system $p(N)$, since $p(N) \sum b_{i}^{*}$ will be constant. This is a pure implication of the public monitoring technology (see footnote 12).

As, on the one hand, the Nash equilibrium of the minimal one-rank extension has this nice feature, on the other hand, it displays a large risk of intemal corruption. If the subordinates could persuade the supervisor to stop monitoring, each would reap the bribe $z$ without any risk. Hence, each subordinate would be willing to "bribe" the supervisor up to $z$, which yields $(N-1) z$, summing over the subordinates. The no-collusion constraint is therefore

$p \mu\left(m^{*}\right) \sum_{i} b_{i}^{*}-c\left(m^{*}\right) \geq(N-1) z$

To eliminate the possibility of internal corruption, the supervisor's expected net gain from monitoring must exceed $(N-1) z$. If (13) does not hold, the worst happens: all $N$ officers are bribed, and the $N-1$ subordinates bribe the supervisor, generating full internal and external corruption. Failure to take into account the possibility of collusion in designing organizations can thus be fatal, a message that recurs as well in the other hierarchies that follow. Since the right-hand side of (13) is increasing in the size of the population, while the left hand side is constant, one can define a critical population size $N^{C}$ beyond which (13) fails to hold. ${ }^{12}$ Proposition 3 summarizes this discussion.

Proposition 3. Under public monitoring in the minimal one-rank extension, the expected per-subordinate level of type-1 equilibrium corruption $\sum_{i} b_{i}^{*} /(N-$ 1) decreases in $N$. This holds as long as $N$ does not exceed a critical bound $N^{C}$ beyond which the outcome switches to collusion.

From (12), and recalling that $m^{*}$ is determined through (4) and hence is independent of $N$, it follows that $\sum b_{i} /(N-1)$ will be nonincreasing in $N$, as mentioned in Proposition 3, even under a general reward structure $p(N)$ per reported bribe, as long as $p(N)$ is not quickly increasing in $N$.

The possibility of bribing one's supervisor is obviously also related to the objective function or type of the supervisor. The necessity of having a "hero" on top of the hierarchy to combat widespread corruption is quite often emphasized in the informal literature. Though the definition or the objective function of a

12. It is worth noting that (13) is not affected by the reward structure. If, more generally, $p$ is replaced by a function $p(N)$ of the population size, the whole impact of modifying $N$ will, in a type-1 equilibrium, be absorbed by $\sum b_{l}$, and $m^{*}$ will remain constant. Evidently, since $p(N) \sum b_{i}$ and $m^{*}$ are constant, there will be no change in the no-collusion constraint (13). 
hero in that literature is not clear, it certainly is not given by (1). If a hero is someone who rejects the bribe $z$ and does not collude with his subordinates, having such a person on top of the minimal one-rank extension in this model may reduce the level of expected bribe incidences from $N$ to $b_{B}^{*}$, which is even less than one! Klitgaart (1991) reports the accomplishments of such a hero at the head of the Philippines Bureau of Internal Revenue.

\subsection{The Maximal One-Rank Extension}

While the minimal one-rank extension is the most centralized monitoring structure, the maximal one-rank extension is the most decentralized, in the sense that it displays the maximum number of independent segments. A feature of the latter extension simplifies the analysis considerably. If $N$ is even, it does not matter whether the monitoring technology is public or private, because each supervisor monitors exactly one subordinate. ${ }^{13}$ Each supervisor-subordinate pair forms an independent unit of analysis, thus, the results derived for the benchmark hierarchy in Section 2 can be applied here in a straightforward way. Letting $\Pi^{m}$ denote the sum of Nash equilibrium bribe acceptance probabilities, it follows that $\Pi^{m}=(N / 2)\left(1+b_{B}^{*}\right)$. As for the potential for internal corruption, note that (13) implies (6): a collusive outcome in the maximal one-rank extension implies a collusive outcome in the minimal one-rank extension. This should not be surprising, in light of the previous result that the likelihood of internal corruption is increasing in the number of monitored subordinates. I compare below the maximal one-rank extension with the minimal one-rank extension under public monitoring.

\section{Proposition 4.}

(i) If $N \leq N^{C}$, the outcome in both extensions is described by the corresponding Nash equilibrium. This yields $(N / 2) \Pi_{m}=\Pi^{m}$ : the number of bribes under the maximal one-rank extension is expected to be $N / 2$ times higher than under the minimal one-rank extension.

(ii) If $2 \leq N^{C}<N$, the outcome is described by the Nash equilibrium in the maximal extension, but the minimal one-rank extension exhibits collusion. Clearly, $\Pi_{m}>\Pi^{m}$.

(iii) If $N^{C}<2$, the outcome is collusive in both extensions. Thus, $\Pi_{m}=$ $\Pi^{m}=N$.

Proof. If (13) holds, the equilibrium monitoring effort $m^{*}$ and bribing strategies are determined by (4) and (5) for the maximal extension, and by (4) and (12) for the minimal extension case. As mentioned in the text, $m^{*}$ is identical in both cases, and $\sum_{i} b_{i}^{*}=b_{B}^{*}$ follows from (5) and (12). The result in (i) follows from the comparison of the expressions for $\Pi_{m}$ and $\Pi^{m}$. If (13) does not hold, but (6) does, that is, if $z \leq p \mu\left(m^{*}\right) \sum_{i} b_{i}^{*}-c\left(m^{*}\right)<(N-1) z, \Pi^{m}$ is

13. If $\mathrm{N}$ is odd, one of the supervisors may have to monitor two subordinates; hence, the monitoring technology will matter. The impact vanishes if $N$ is large. I assume that $N$ is even in the discussion of the maximal one-rank extension. 
as given above, but the outcome in the minimal one-rank extension is collusive, so $b_{i}=1$ for all $i$ and $\Pi_{m}=N$. Finally, if (6) does not hold for $N \geq 2$, it follows that $b_{i}=1$ in both cases; hence, $\Pi_{m}=\Pi^{m}=N$.

With public monitoring, the Nash equilibrium of the minimal one-rank extension exhibits lower corruption than the maximal one-rank extension. However, the scope for intermal comption is larger in the minimal one-rank extension, as (13) is more restrictive than (6); we may well be in case (ii) of Proposition 4 with $\Pi_{m}=N$. In such a case, provided that $\Pi^{m}<N$, an "intermediate" one-rank extension will be the best one-rank extension. One can build such an intermediate one-rank extension by dividing the population into a minimum number of independent groups, such that the size of each group is smaller than $N^{C}$, and then forming the minimal one-rank extension of each group. For example, if $N^{C}<N \leq 2 N^{C}$, one can form two independent groups of sizes $N_{1} \leq N^{C}$ and $N_{2} \leq N^{C}$, then minimally extend each group by one rank. This generates two independent segments, each being a minimal one-rank extension hierarchy. The supervisor of one group monitors $N_{1}-1$ subordinates, while the other monitors $N_{2}-1$. I call this structure a two-group one-rank extension. More generally, depending on the value of $N^{C}$, one can form $G$ independent segments from the population, if necessary, and then minimally extend each segment by one rank. The resulting hierarchical structure will be called the $G$-group one-rank extension of the population.

Proposition 5. Given the incentive system $(w, p)$ and the public monitoring technology, corruption is minimized under the $G$-group one-rank extension, where $G$ is the smallest integer satisfying $N^{C} \geq N / G$.

The hierarchy described in Proposition 5 minimizes the expected number of bribes $G\left(1+b_{B}^{*}\right)$ accepted in the population by minimizing $G$. The constraint is that in each group $j, N_{j} \leq N^{C}$; the number of subordinates should be lower than $N^{C}$ to check for the hazard of internal corruption. Increasing the number of ranks or modifying the number of independent groups will only increase the expected level of corruption. ${ }^{14}$

14. The result in Proposition 5 is also likely to hold under an endogenized incentive system $\left[w_{i}, p(N)\right]$, because its driving force is economies of scale in monitoring. The reward system should clearly respond to these economies of scale that favor the minimal one-rank extension. Though the critical population size $N^{C}$ surely exists as an upper bound beyond which the outcome becomes collusive, note that $N^{C}$ will also be affected by the incentive system. The optimal number of independent one-rank extensions will then depend on the trade-off between corruption and the cost of monetary incentives, as dictated by the objective of the principal. This further complicates the analysis of the endogenous incentives case. Intuition suggests, however, that as the population size increases, a size will be reached beyond which the option of breaking up the population into two independent one-rank extensions will dominate the option of continually adjusting economic incentives to increase $N^{C}$ in order to keep internal corruption under control. 


\section{Private Monitoring}

\subsection{The Minimal One-Rank Extension}

Since I assumed a type-1 equilibrium in the benchmark hierarchy, the Nash equilibrium in the maximal one-rank extension will also be of type 1 , no matter whether monitoring is private or public. However, in the minimal one-rank extension, the monitoring technology makes a big difference as to the equilibrium level of comption. In fact, the Nash equilibrium under private monitoring moves towards a type- 2 equilibrium as the number of subordinates per supervisor is increased, because total monitoring costs increase sharply due to the strict convexity of the cost function. The monitoring effort of the supervisor tends to decrease, and subordinates are more inclined to accept the bribes.

To begin, consider the symmetric, type-1 Nash equilibrium where each subordinate is monitored with the same effort $m^{*}$, generating the same bribe acceptance strategy $b_{i}^{*}$ for each subordinate $i$. Hence, a type-1 equilibrium, if an interior solution $b_{i}^{*} \in(0,1)$ exists, is described by (4) and

$$
p b_{1}^{*}=\frac{\partial c\left[(N-1) m^{*}\right] / \partial m}{\partial \mu\left(m^{*}\right) / \partial m} .
$$

Since the total cost of monitoring $N-1$ subordinates, $c[(N-1) m]$, is strictly convex and increasing, the supervisor may optimally choose a lower monitoring effort $m<m^{*}$, possibly leading to a type-2 Nash equilibrium. Such an equilibrium involves $b_{i}^{*}=1$ and (14) holding with equality for $b_{i}^{*}=1$. However, even if the equilibrium is of type 1 , comparing (14) with (5) and using the assumption of rising marginal costs reveals that $b_{i}^{*} \geq b_{B}^{*}$, that is, the subordinates put more weight on bribe acceptance as the number of peers increases. Under private monitoring, larger teams of monitored subordinates should be expected to exhibit more external corruption. Using (5) and (14) in $\Pi_{m}=1+(N-1) b_{i}^{*}$ and $\Pi^{m}=(N / 2)\left(1+b_{B}^{*}\right)$, it can be shown that $\Pi_{m}$ and $\Pi^{m}$ depend on, besides the parameters of the model, the curvatures of $c(\cdot)$ and $\mu(\cdot)$. Though in the minimal extension the number of subordinates is higher $(N-1>N / 2)$, and each subordinate is more likely to accept the bribe $\left(b_{i}^{*}>b_{B}^{*}\right)$, we have more supervisors in the maximal extension $(N / 2>1)$ who are not monitored, and hence, who surely accept the bribe. For a sufficiently large $N$, Proposition 6 presents a clear result about the relative corruption in the Nash equilibria of these two polar hierarchies.

Proposition 6. Under private monitoring and the incentive system $(w, p)$, if the population size is sufficiently large, the Nash equilibrium outcome of the minimal one-rank extension is expected to display higher corruption than the maximal one-rank extension.

Proof. Since $b_{t}^{*}>b_{B}^{*}$, the equilibrium must be of type 2 in the minimal extension, if the equilibrium is of type 2 in the maximal one-rank extension. Therefore, consider a type- 1 equilibrium in the maximal extension and assume $0<b_{B}^{*}<b_{i}^{*}<1$. By strict convexity of $c(\cdot)$, a population size $\bar{N}+1$ must exist such that $p\left[\partial \mu\left(m^{*}\right) / \partial m\right]<\partial c\left(\bar{N} m^{*}\right) / \partial m$, but $\left[\partial \mu\left(m^{*}\right) / \partial m\right] p b_{i}^{*}=$ 
$\partial c\left((\bar{N}-1) m^{*}\right) / \partial m$ where $b_{i}^{*}<1$, and $m^{*}$ satisfies (4). Hence, $\Pi_{m}(1)=N$ for $N>\bar{N}$, while $\Pi^{m}(1)<N$ holds for all $N$.

Because of rapidly rising monitoring costs, equilibrium monitoring decreases and corruption increases as the population, and thus the number of subordinates, gets larger. There will be a critical population size $\bar{N}+1$ beyond which the Nash equilibrium of the minimal one-rank extension switches from type 1 to type 2: all $\bar{N}$ subordinates set $b_{i}^{*}=1$. Therefore, given the incentive system $(w, p)$, the maximal one-rank extension is surely less corrupt than the minimal one-rank extension, if the population is large.

Now, include the possibility of internal corruption in the picture. The nocollusion constraint is again (6) for the maximal one-rank extension, and

$\mu\left(m^{*}\right) \sum_{i} b_{i}^{*} p-c\left[(N-1) m^{*}\right] \geq(N-1) z$

for the minimal one-rank extension. Remember, (15) is obtained from the supervisor's objective through the condition that the maximum the subordinates are willing to pay to avoid being monitored should not exceed the expected net rewards from monitoring $N-1$ subordinates. Comparing (15) with (6) does not yield a clear-cut result on which structure is more likely to display internal corruption. But the fact that (15) depends on the size of the population, while (6) does not, provides a generalization of Proposition 6: Under the incentive system $(w, p)$, expected level of corruption may be lower in the minimal onerank extension than the maximal one-rank extension, only if the population size is small.

The analysis above involves pairwise comparisons of two extreme one-rank extensions of the population. To obtain a ranking of two-rank hierarchies for all $N$ under private monitoring, one has to compare quite a large number of corresponding equilibria. ${ }^{15}$ Instead, I focus below on the most decentralized two-rank hierarchy (the maximal one-rank extension). The analysis will confirm the intuition that the curvatures of $c(\cdot)$ and $\mu(\cdot)$ play an important role, and it will also provide some insight as to the role of other parameters, such as $p$, in determining the ranking of two-rank hierarchies, in terms of their expected corruption levels. As the analysis above suggests, the private monitoring technology favors rather steep hierarchies, possibly involving independent segments. I present below a necessary condition under which the maximal one-rank extension displays the lowest expected comuption. Let $N=3$. Figure 3 displays the two possible one-rank extensions in a population of three. In case $A$, only one agent is monitored, while in case $B$, one supervisor monitors the other two agents. I introduce the notation $b_{i}^{(k) *}$ for the Nash bribe acceptance strategy of

15. For instance, in the case of $N=4$, besides (i) the minimal and (ii) the maximal one-rank extensions, there are two other hierarchies: (iii) the minimal one-rank extension of the group of three agents, leaving the fourth not monitored; and (iv) the case where one agent monitors another, while the remaining two are not monitored. Even in this simple case, the only straightforward conclusion is that hierarchy (iii) dominates hierarchy (iv). The ranking of hierarchies (i), (ii) and (iii) depends on the form of $c(\cdot)$ and $\mu(\cdot)$. 


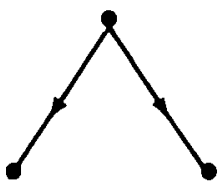

Case B

Figure 3. Two hierarchical organizations with three agents.

a subordinate in a one-rank minimal extension consisting of $k$ agents, $k-1$ of which are subordinates. Note that in a population of two agents, $b_{i}^{(2) *}=b_{B}^{*}$. Finally, let $\Pi^{A}$ and $\Pi^{B}$ be the sum of equilibrium bribe acceptance probabilities in cases $A$ and $B$. The following proposition states that if case $B$ exhibits higher corruption than case $A$, then expected corruption is minimal in the maximal one-rank extension among all possible two-rank hierarchies.

Proposition 7. $\Pi^{A}<\Pi^{B}$ implies $\Pi_{m}<\Pi^{j}$ for all $N$ and any one-rank extension $j$.

Proof. $\Pi^{A}<\Pi^{B}$ can be written as $1+b_{B}^{*}<2 b_{i}^{(3) *}$. Note that all one-rank extensions can be viewed as combinations of minimal and maximal one-rank extensions of subgroups of the population. Therefore, if $\Pi_{m}<\Pi^{m}$ for all $N$, then $\Pi_{m}<\Pi^{j}$ for any one-rank extension $j$. It is thus sufficient to prove that $1+b_{B}^{*}<2 b_{i}^{(3) *}$ implies $\Pi_{m}<\Pi^{m}$ for all $N$. But this follows in two steps: (i) recall that $b_{i}^{(N) *}$ is increasing in $N$, and (ii) in the minimal one-rank extension, $\Pi_{m}=1+(N-1) b_{1}^{(N) *}$, while in the maximal one-rank extension, $\Pi^{m}=(N / 2)\left(1+b_{B}^{*}\right)$, if $N$ is even, and $\Pi^{m}=1+[(N-1) / 2]\left[1+b_{B}^{*}\right]$, if $N$ is odd. Thus, for even $N,(N / 2)\left(1+b_{B}^{*}\right)<N b_{i}^{(3) *}<N b_{i}^{(N) *} \leq 1+(N-1) b_{i}^{(N)^{*}}$, and for odd $N,[(N-1) / 2]\left[1+b_{B}^{*}\right]<(N-1) b_{i}^{(3) *}<(N-1) b_{i}^{(N)^{*}}$, which completes the proof.

The condition $\Pi^{A}<\Pi^{B}$, that is, $1+b_{B}^{*}<2 b_{i}^{(3) *}$, can be expressed as

$p \frac{\partial \mu\left(m^{*}\right)}{\partial m}+\frac{\partial c\left(m^{*}\right)}{\partial m}<2 \frac{\partial c\left(2 m^{*}\right)}{\partial m}$

through substitutions from (5) and (14). Inequality (16) shows that $\Pi^{A}<\Pi^{B}$ is likely to hold if $p$ is low, marginal costs are increasing sufficiently quickly, or monitoring success is inelastic in effort. This is intuitive, because a low $p$ or rapidly increasing monitoring costs can reduce monitoring incentives more in case $B$ than they do in case $A$. Otherwise, if (16) does not hold, under private monitoring, the minimal (or an intermediate) one-rank extension may yield a lower expected corruption level than the maximal one-rank extension. 


\subsection{Private Monitoring in a Population of Three}

Two possible ways of organizing three agents in a hierarchy are shown in Figure 3 and compared in Proposition 7. The third possible structure is the supervision chain studied in Section 3. Comparing the Nash equilibrium outcomes reveals that the expected level of corruption is lower in the three-rank hierarchy than the hierarchy in case $A$ of Figure 3 , as $1+b_{2}^{*}+b_{3}^{*}<2+b_{B}^{*}$. Furthermore, if (16) holds so that $2+b_{B}^{*}<1+2 b_{i}^{(3) *}$, case $A$ should be preferred to case $B$. Therefore, under the incentive system $(w, p)$ with private monitoring and $N=3$, the three-rank hierarchy yields the lowest expected corruption level. Recall the requirements of (16): $p$ must be sufficiently small and/or the function $c(\cdot)$ must be sufficiently convex (rapidly rising monitoring costs). Under these conditions, corruption-minimizing hierarchies should have a quite steep outlook.

\section{Concluding Remarks}

For a variety of reasons, including enforceability of contracts, unclear organizational goals, and lack of relevant preformance statistics, comupt behavior can hardly be detected unless appropriate monitoring technologies are implemented. This article focuses on the monitoring function of hierarchies. Given the pattern of supervision implied by the hierarchical structure, it addresses the question of what hierarchical structure minimizes corruption in a population of identical agents who receive the same wage with a reward $p$ per successful bribe report for the supervisors. The article presents a basic model of individual members' behavior and examines different hierarchical forms. The model allows for both external and internal dimensions of corruption and identifies a trade-off between the two.

The analysis shows that the ranking of hierarchies in terms of displayed corruption depends heavily on the technology of monitoring, that is, whether and how monitoring costs are affected by the number of monitored subordinates. When monitoring costs show no dependence on the number of monitored supervisors, each supervisor should optimally monitor as many subordinates as possible. But the no-collusion constraint imposes an upper bound $N^{C}$ on this number. If this constraint is binding, the hierarchy must involve structurally independent segments, such that the number of independent segments is determined by the highest integer smaller than the ratio $N / N^{C}$ in a population of $N$. On the other hand, under private monitoring, obtaining a complete ranking of hierarchies in terms of corruption seems to be a difficult task. Alternative hierarchical structures proliferate quickly as $N \geq 4$, making it impossible to compare most pairs of structures without specific assumptions about $c(\cdot)$ and $\mu(\cdot)$. However, strict convexity of monitoring costs is clearly a factor favoring steep hierarchies. If monitoring costs are sufficiently convex, the expected level of corruption would be minimized by assigning a supervisor to not more than one subordinate. The hierarchy would then consist of $N$ ranks, that is, a supervision chain of $N$ agents.

I close the article with some extensions for future research in addition to those mentioned in Section 2.2. The setting in this article is static. It is well 
known that in repeated games, "cooperation," which in this paper would take the form of internal corruption, can be supported as a subgame-perfect equilibrium outcome if the parties are sufficiently patient and farsighted. An extension of, for instance, the benchmark model to a repeated game setting can therefore provide insights about the role of organizational measures to combat corruption in a dynamic perspective. By committing himself to a probability of rotating the agents among various hierarchical positions, the principal or the designer of an organization can affect the discount factors of the members, hence indirectly keeping intemal comuption under control. The incentive system can be focused exclusively on minimizing external corruption, while the rotation policy checks for the hazard of internal corruption. In practice, occupational mobility in organizations serves as a device to stop "excessive friendship" and nonmonetary transfers among the members. Examples are rotations in the diplomatic corps and the civil service. The cost of rotating the members, also recognized by Tirole (1988), would be the loss of beneficial cooperation, an aspect that I ignored in this article. The extension to a repeated game will provide a formal framework in which optimal rotations and durations of hierarchical occupations can formally be studied.

Extending the model to allow for both peer monitoring and hierarchical monitoring will raise a number of interesting and unresolved questions. How do these two monitoring modes interact? Do they complement or substitute each other? In the absence of internal corruption, intuition suggests that peer monitoring can be an effective way of controlling corruption in flat hierarchies, when monitoring costs increase quickly in the number of subordinates. Partial delegation of the monitoring to the monitored may reduce monitoring costs and increase effectiveness, but it may also affect the possibilities for internal comuption. However, this conjecture awaits being addressed in a formal model.

\section{References}

Alchian, Armen, and Harold Demsetz. 1972. "Production, Information Costs and Economic Organization," 75 American Economic Review 777-95.

Bac, Mehmet. 1996. "Corruption and Supervision Costs in Hierarchies," 22 Joumal of Comparative Economics 99-118.

Basu, Kaushik, Sudipto Bhattacharya, and Ajit Mishra. 1992. "Notes on Bribery and the Control of Comption," 48 Joumal of Public Economics 349-59.

Baron, David P., and David Besanko. 1992. "Information, Control and Organizational Structure," 1 Journal of Economics and Management Strategy 237-275.

Bolton, Patrick, and Mathias Dewatripont. 1994. "The Firm as a Communication Network," 109 Quarterly Journal of Economics 809-39.

Calvo, Guillemo A. 1987. "The Economics of Supervision," in H. Nalbantian, ed., Incentives, Cooperation and Risk Sharing. New Brunswick: Rowman \& Litulefield.

Calvo, Guillermo A., and Stanislaw H. Wellisz. 1978. "Supervision, Loss of Control, and the Optimal Size of the Firm," 86 Journal of Political Economy 943-52.

Cyert, Richard M., and James G. March. 1963. A Behavioural Theory of the Firm. : Prentice Hall.

Har, Oliver, and Bengt Holmström. 1987. "The Theory of Contracts," in T. Bewley, ed., Advances in Economic Theory, Fifth World Congress. Cambridge: Cambridge University Press.

Jabbra, Jamaal G. 1989. "Public Sector Accountability in the Arab World: The Cases of Lebanon, 
Egypt and Saudi Arabia," in J. G. Jabbra and O. P. Dwivedi, ed, Public Sector Accountability. Hartford: Kumarian Press.

Klitgaart, Robert. 1991. "Gifts and Bribes," in R. J. Zechauser, ed., Strategy and Choice. Cambridge: MIT Press.

Major, Ivan. 1993. Privatization in Eastern Europe. Hants: Edward Elgar Publishing.

Myerson, Roger B. 1993. "Effectiveness of Electoral Systems for Reducing Government Corruption: A Game-Theoretic Analysis," 5 Games and Economic Behavior 118-32.

Pechlivanos, Lambros. 1995. "Sustainability of Comuption and Optimal Deterrence." Presented at the Seventh World Congress of Econometric Society, Tokyo, Japan.

Radner, Roy. 1993. "The Organization of Decentralized Information Processing," 61 Econometrica $1109-46$.

Shleifer, Andrei, and Robert W. Vishny. 1993. "Corruption," 108 Quarterly Journal of Economics 519-617.

Tirole, Jean. 1986. "Hierarchies and Bureaucracies: On the Role of Collusion in Organizations," 2 Joumal of Law, Economics, \& Organization 181-214.

1988 "The Multicontract Organization," 21 Canadian Journal of Economics 459-66.

Varian, Hal R. 1990. "Monitoring Agents with Other Agents," 146 Journal of Institutional and Theoretical Economics 153-74.

Williamson, Oliver. 1967. "Hierarchical Control and the Optimal Firm Size," 75 Joumal of Political Economy 123-38. 